\title{
Significant Improvement of Straw Lignocellulose Degradation Rate by Subordination Consortium Composed of Cellulomonas ZJW-6 and Acinetobacter DA-25
}

\author{
Yunpeng Guan \\ Jilin Agricultural University \\ Hongyu Zhu \\ Jilin Agricultural University \\ Yuan Zhu \\ Jilin Agricultural University \\ Longhua Shu \\ Jilin Agricultural University \\ Hemei Zhao \\ Jilin Agricultural University \\ Jian Song \\ Jilin Agricultural University \\ Xue Yang \\ Jilin Agricultural University \\ Zhihai Wu \\ Jilin Agricultural University \\ Lei Wu \\ Jilin Agricultural University \\ Meiying Yang ( $\nabla$ jlaumeiying@163.com ) \\ https://orcid.org/0000-0003-1295-5472
}

\section{Research}

Keywords: Lignocellulose, microbial consortium, Cellulomonas, Acinetobacter, enzyme activity complementation, induction test

Posted Date: June 25th, 2021

DOI: https://doi.org/10.21203/rs.3.rs-629481/v1 
License: (c) (i) This work is licensed under a Creative Commons Attribution 4.0 International License. Read Full License 


\section{Abstract}

\section{Background}

The effective utilization of lignocellulose is essential for achieving circular economy and sustainable agricultural development. The screening of lignocellulose-degrading strains from the environment and artificial establishment of microbial consortia can achieve better degradation effects. In this paper, microbial consortium 625 was established and screened based on high lignocellulose degradation ability but low $\beta$-glucosidase activity of Cellulomonas ZJW- 6 to complement enzyme activity and enhance lignocellulose degradation by exerting the subordination effect. This research provides an effective strategy for artificially establishing microbial consortia, improving lignocellulose degradation efficiency, and enhancing bioenergy production.

\section{Results}

In order to establish high-efficiency microbial consortia, Cellulomonas ZJW-6 with low $\beta$-glucosidase activity was combined in pairs with 14 strains with high $\beta$-glucosidase activity according to the enzyme activity complementation principle, from which microbial consortium 625 composed of strains ZJW-6 and DA-25 was screened, which had significantly enhanced lignocellulose degradation rate compared with purely cultured ZJW- 6 strain and other 13 combinations. The single factor test showed that consortium 625 had better $\mathrm{pH}$ and temperature tolerance. The lignocellulose degradation rate of this consortium was $57.62 \%$ after verification test under optimized conditions using response surface method. The induction test showed that the growth of strain DA-25 was inhibited by strain ZJW- 6 and its filtrate, while strain DA-25 and its filtrate promoted the growth of strain ZJW- 6 and significantly enhanced the activities of key enzymes in the consortium 625 lignocellulose degrading system, among which the $\beta$ Glucosidase activity was significantly higher than that in purely cultured ZJW-6 strain.

\section{Conclusion}

Microbial consortium 625, composed of Cellulomonas ZJW-6 (dominant strain) and Acinetobacter DA-25, with high lignocellulose degradation efficiency, was screened according to the enzyme activity complementation principle. Interestingly, microbial consortium 625 exhibited complementation in enzyme activities of its two strains, improvement in its tolerance to temperature and $\mathrm{pH}$, and the same effect of DA-25 filtrate as strain DA-25. Based on the previous studies on Acinetobacter, we speculate that a certain compound adverse to the growth of strain DA-25 may be produced during lignocellulose degradation by strain ZJW-6, while secretions of strain DA-25 inhibit this compound leading to an increase in the growth and enzyme activity of strain ZJW-6.

\section{Background}

Straw (lignocellulose) is a highly complex heterogeneous polymer produced by plant photosynthesis, mainly composed of cellulose, hemicellulose, and lignin [1,2]. Lignin wraps cellulose and hemicellulose 
forming a tight physical barrier, which hinders the degradation of lignocellulose [3]. However, the effective utilization of this substance is essential for achieving circular economy and sustainable agricultural development [4].

Abundant lignocellulose-degrading microorganisms exist in the natural environment, mainly including bacteria and fungi, which are numerous in variety. Currently, more than 200 purely cultured microorganisms have been isolated from compost, rumen and anaerobic sludge [5]. Fungi are characterized by high degradation efficiency, extensive functions, etc. mainly including white-rot fungus [6], aspergillus [7] and penicillium [8]. However, their applications are limited due to the pathogenicity of some fungi. Bacteria have high reproducibility, acid-alkali resistance, etc. Lignocellulose-degrading bacteria mainly include pseudomonas [9], bacillus [10], caldicellulosiruptor bescii [11] and clostridium [12]. However, their degradation ability is more unitary [13]. Most bacteria are only able to degrade substrates with simple structure, and microorganism cultivation conditions are very different from each other, so natural lignocellulose cannot be effectively used [14].

It should be noted that the degradation of straw in nature does not depend entirely on certain microorganisms, but completed by mutual effects and interactions of microbial communities in soil. Therefore, many scholars have conducted a lot of research on mixed microorganisms regarding their degradation of lignocellulose. Experiments have demonstrated that the ability of mixed microorganisms to degrade straw is stronger than any single strain $[15,16,17]$. The screening of natural microbial consortia from the environment and artificial establishment of microbial consortia by simulating natural conditions have both achieved better degradation effects. After long-term soil treatment, Guo et al. screened an effective natural lignocellulose-degrading microbial consortium XDC-2 from compost and soil amended with animal manure, which has a degradation rate of $60.3 \%$ for rice straw and wide $\mathrm{pH}$ adaptability [18]. Research conducted by Liang et al. showed that a flora was inoculated into a medium with lignocellulose and chlophenol as carbon sources for domestication and culture to obtain a microbial consortium (OEM2) with chlophenol detoxification and lignocellulose deconstruction capabilities after subculture, which has a degradation rate of $41.5 \%$ for $12 \mathrm{~d}$ rice straw [19]. Pilar et al. (2018) established 65 microbial consortia with screened 18 strains representing four different metabolic functions according to the complementary principle. The results showed that the degradation efficiency of the established microbial consortia has been significantly improved [20]. Wang et al. obtained microbial consortium WCS- 6 through continuous subculture by taking straw as a substrate, with a lignocellulose degradation rate of up to $80 \%$ [21]. Lu et al. screened 15 strains with higher cellulase activity from flower stemvegetable wastes, and combined them into four groups of mixed cultures with significantly enhanced cellulase activity compared with single strains [22].

In earlier period, the research group took samples at several different locations and selected 27 bacterial strains with lignocellulose degradation ability, among which ZJW-6 had a significantly higher degradation rate than that of other strains, but the $\beta$-glucosidase activity of this strain was found to be low during the research process. Considering that $\beta$-glucosidase is the key enzyme during lignocellulose degradation [23], we took strain ZJW-6 as the main subject and respectively established microbial consortia by 
combining ZJW- 6 and other 14 strains with higher $\beta$-glucosidase activity based on the principle of enzyme activity complementation. The results showed that the combination of ZJW-6 and DA-25 exhibited higher lignocellulose degradation ability and enzyme activity. This research provides an effective strategy for artificially establishing microbial consortia, improving lignocellulose degradation efficiency, and

\section{Results}

\section{Comparison of lignocellulose degradation rate of microbial consortia}

Taking strain ZJW- 6 as the main test strain, 14 strains with higher $\beta$-glucosidase activity (Fig.S1) were screened and combined into 14 microbial consortia according to the principle of enzyme activity complementation. The lignocellulose degradation rate of the 14 microbial consortia is shown in Fig. 1. The results showed that the degradation rate of the microbial consortium 625 composed by ZJW- 6 and DA-25 was highest (53.2\%), which was significantly higher than the degradation rate (49.6\%) of single strain ZJW-6. However, the degradation rate of the other 13 microbial consortia showed a decline, which was significantly lower than that of single strain ZJW-6.

\section{Results of 16srRNA identification of strains DA-25 and ZJW- 6}

Strain ZJW-6 belongs to the genus Cellulomonas (data not shown). In order to determine the characteristics of strain DA-25 in consortium 625, the strain was identified using the 16S rDNA sequencing method. The $16 \mathrm{~S}$ rDNA sequences of strain DA-25 were compared with the 16S rDNA sequences of some sequenced Acinetobacter strains in the NCBI database. Taking Cellulomonas sp.strain S5b8 and strain ZJW-6 as the outgroup, a phylogenetic tree was built with MAGE X 10.2.5. The results are shown in Fig. 2. Strain DA-25 and Acinetobacter strains form a branch. Therefore, strain DA-25 belongs to the genus Acinetobacter, with a support rate of $97 \%$.

\section{Optimization of degradation conditions for microbial consortium 625}

\section{Results of single factor test}

In order to determine the maximum potential of microbial consortium 625 for degradation of lignocellulose, the degradation conditions were optimized. The single factor test showed that the degradation rate was the highest under the conditions of $8 \mathrm{~g} / \mathrm{L}$ peptone as a nitrogen source, inoculation ratio (3:1) of strain ZJW- 6 to DA-25 (Fig.S2), $\mathrm{pH}=9$, and $31^{\circ} \mathrm{C}$ culture temperature. Therefore, the subsequent response surface tests were conducted under the above conditions. 
Meanwhile, the tolerance range of $\mathrm{pH}$ and temperature of consortium 625 for lignocellulose degradation was also significantly improved compared to single strain ZJW-6 (Fig. 3). The lignin degradation rate of strain ZJW- 6 was slightly higher than $50 \%$ at $\mathrm{pH} 7-9$, but the degradation rate of consortium 625 also exceeded $50 \%$ at $\mathrm{pH} 6$. Especially at $\mathrm{pH} 5$, the degradation rate of strain ZJW- 6 was less than $20 \%$, but the degradation rate of consortium 625 could reach $46.9 \%$, and the same at $\mathrm{pH} 10$. The optimal degradation temperature of both single strain ZJW- 6 and consortium 625 was $31^{\circ} \mathrm{C}$, but the degradation rate of strain ZJW- 6 was lower than $50 \%$ under other temperature conditions, while that of consortium 625 at $28-37^{\circ} \mathrm{C}$ was significantly higher than 50

\section{ZJW-6 + DA-25 response surface analysis}

The results of the interaction between nitrogen addition amount and $\mathrm{pH}$ indicate that $\mathrm{pH}$ had a greater impact on the degradation rate (Fig. 4A). The contribution of nitrogen addition amount to the degradation rate was equivalent to that of temperature to the degradation rate (Fig. 4B). The results of the interaction between nitrogen addition amount and inoculation ratio indicate that the interaction between the two had a significant impact on the degradation rate (Fig. 4C). The results of the interaction between $\mathrm{pH}$ and temperature indicate that the optimal degradation rate could be obtained at the critical condition of around $\mathrm{pH}$ 8.5. The critical temperature for the optimal degradation rate was $31^{\circ} \mathrm{C}$ when it changes with temperature (Fig. 4D). The results of the interaction between $\mathrm{pH}$ and inoculation ratio exhibited a cambered surface, and the effects of $\mathrm{pH}$ on the degradation rate showed a trend of first increasing and then decreasing, while the change of inoculation ratio had no significant impact on the degradation rate (Fig. 4E). The results of the interaction between temperature and inoculation ratio indicate that the interaction between the two had a significant impact on the degradation rate (Fig. 4F).

In order to analyze the effects of the synergistic interaction between various factors on the degradation rate, further determine the global optimal solution, and achieve the optimization target of maximum degradation rate, the results were analyzed with Design-Expert 10.0.3, showing that the degradation rate predicted by the model was $57.80 \%$ under the conditions of $8.36 \mathrm{~g} / \mathrm{I}$ nitrogen addition, $8.58 \mathrm{PH}, 30.55^{\circ} \mathrm{C}$ temperature, and 2.80:1 inoculation ratio. The experiment of the model prediction results under the conditions of $8.36 \mathrm{~g} / \mathrm{l}$ nitrogen addition, $8.6 \mathrm{pH}, 30.6^{\circ} \mathrm{C}$ temperature, and $2.8: 1$ inoculation ratio showed that the degradation rate of lignocellulose was $57.62 \%$, which was close to the model prediction results.

\section{Determination of growth rate of strains in consortium 625 and induction test}

In order to explore the growth of strains ZJW- 6 and DA-25 in the consortium 625 co-culture system, the growth rate of the strains within $96 \mathrm{~h}$ was determined (Fig. 5), showing that the co-culture did not affect the growth of strains ZJW-6 and DA-25 compared with the mono-culture of them, and the time required for growth to reach the plateau was also unchanged. However, the growth of strain ZJW-6 in the coculture system was significantly promoted, and the growth of the strain at the logarithmic phase within 24-96h was significantly higher than that in the mono-culture system. On the contrary, the growth of 
strain DA-25 in the co-culture system was significantly inhibited, with a lower growth rate than that under mono-culture conditions.

To determine whether this growth of strains ZJW- 6 and DA-25 in consortium 625 was due to direct contact between bacteria or induction of bacterial secretions, an induction test was carried out. In the induction test, the growth of strain ZJW- 6 added with DA-25 filtrate was significantly promoted, which was consistent with the growth of strain ZJW-6 in consortium 625 (Fig. 5A). On the contrary, the growth of strain DA-25 added with ZJW-6 filtrate was inhibited, also showing the same growth state as in consortium 625 (Fig. 5B). Therefore, we determined that the growth of strain ZJW-6 in consortium 625 was promoted by DA-25 secretion, while the ZJW- 6 filtrate inhibited the growth of strain DA- 25 .

\section{Changes in the activity of key lignocellulose degradation enzymes}

Figure 6 shows the activity determination results of key enzymes for degrading lignocellulose in consortium 625 and induction test. Figure $6 \mathrm{C}$ indicates the determination result of $\beta$-Glucosidase activity, showing that the $\beta$-Glucosidase activity in consortium 625 was significantly affected by strain DA-25, and significantly higher than the activity of the enzyme in single strain ZJW-6. The results of the induction test were consistent with those of consortium 625 , and the activity of the enzyme in the induction system of strain ZJW-6 added with DA-25 filtrate was also significantly enhanced. Figures 6A-6B and 6D-6F indicate that, the activity of endoglucanase, xylanase, laccase and lignin peroxidase showed the similar law: the activity of enzymes in the consortium 625 degradation system was significantly affected by DA25 , which was significantly higher than that of each enzyme in the degradation system of single strain ZJW-6, and the activity of enzymes in the induction test by adding DA-25 filtrate was enhanced compared with that of enzymes in the degradation system of single strain ZJW-6. The activity of enzymes in the DA25 induction test by adding ZJW-6 filtrate was significantly lower than the former, approaching the activity of enzymes in the degradation system of single strain DA-25. It shows that consortium 625 composed by strains ZJW- 6 and DA-25 could compensate for the low activity of $\beta$-Glucosidase in strain ZJW- 6 to achieve the complementation of enzyme activity. In addition, the secretions produced by strain DA-25 could activate the activity of enzymes in the process of lignocellulose degradation by strain ZJW6.

\section{Comparison of lignocellulose degradation rate between induction test and ZJW- 6 or consortium 625}

Consortium 625 can enhance the degradation rate of lignocellulose, which has been confirmed in the consortium screening test. In order to determine the effects of DA-25 filtrate on the degradation rate of lignocellulose by promoting the growth of strain ZJW- 6 and increasing enzyme activity in the induction test, we compared the lignocellulose degradation rate obtained in the induction test and that of strain ZJW-6 or consortium 625 . Figure 7 shows that the degradation rate of lignocellulose by the ZJW- 6 degradation system added with DA-25 filtrate was significantly promoted compared with that of single 
strain ZJW-6, but slightly lower than that of consortium 625. However, the degradation system of strain DA-25 added with ZJW- 6 filtrate exhibited the consistence between the growth rate and enzyme activity of the strain, and its degradation rate of lignocellulose was significantly lower than that of single strain ZJW-6 and consortium 625 degradation system.

\section{Discussion}

Lignocellulose is a complex composed of cellulose, hemicellulose and lignin. Cellulose acts as the structural skeleton in the center to provide support. Hemicellulose and lignin wrap cellulose therein and intertwine to form neatly arranged crystalline areas and relatively loose amorphous areas, which are difficult to degrade due to their compact structure[29]. In nature, a variety of microorganisms exist and can hydrolyze lignocellulose into small-molecule monosaccharides by the synergistic action of enzymes, and then ferment them into products that can be used by people. Therefore, the biodegradation and reuse of lignocellulose has become a focus of current research.

Lignin is a complex phenolic polymer with a three-dimensional network formed by three alcohol monomers connected to each other through ether bonds and carbon-carbon bonds. It has oxidizing lignin peroxidase (LiP) (EC1.11.1.13), manganese peroxidase (MnP) (EC1.11.1.14) and laccase (Lac) (EC 1.10.3.2)[30], which is able to oxidize phenolic lignin structures into phenoxy radicals, resulting in decomposition of compounds[31]. Xylan is one of the main components of hemicellulose. It is a complex formed by poly-pentaglucose side chains connected to lignin, pectin and other substances through chemical bonds and hydrogen bonds[32]. Xylanase(EC3.2.1.8) can hydrolyze macromolecular xylan into xylo-oligosaccharide or xylose monomer. Exoglucanase (CBH) (EC3.2.1.91) mainly hydrolyzes $\beta-1$, 4glycosidic bonds by combining the reducing end of crystalline region of cellulose to release cellobiose, so as to gradually break up well-arranged firm crystalline regions. Endoglucanase (EC3.2.1.4) acts on amorphous non-crystalline areas in cellulose to mainly hydrolyze $\beta$-1,4-glycosidic bonds between glucose, releases oligosaccharides, and randomly break cellulose chains to expose more ends of cellulose.

Exoglucanase and endoglucanase work synergistically on cellulose to produce cello-oligosaccharide and cellobiose, and the latter is converted to glucose by $\beta$-glucosidase[33]. The use of glucose produced by microbial enzymatic hydrolysis of lignocellulose to prepare biofuels[34] can effectively reduce the waste of straw resources and our dependence on fossil fuels.

The use of biocomposites is considered to be an effective method to degrade natural lignocellulosic materials [35]. TMC7, a microbial consortium, has a lignocellulose degradation rate of $79.7 \%$ within 15 days under high temperature conditions, which is unmatched by any single strains[36]. The artificial combination of screened single strains to obtain microbial consortia with stronger lignocellulose degradation ability is also feasible and effective. The microbial consortium WCS- 6 established by Wang et al. in 2011 has extremely high degradation efficiency on filter paper, cotton and straws [37]. Cellulomonas ZJW-6 is a high-efficiency lignocellulose-degrading strain obtained in the laboratory. According to the principle of enzyme activity complementation, consortium 625 established by combining screened strain ZJW-6 and Acinetobacter DA-25 can effectively enhance the degradation rate 
of lignocellulose. After conditions are optimized, the degradation rate of lignocellulose by the consortium can reach more than $57 \%$. In addition, the environmental tolerance of consortium 625 is significantly better than that of single strain $\mathrm{ZJW}-6$, and the degradation rate (after $96 \mathrm{~h}$ ) at $\mathrm{pH} 6-9$ or $28-37^{\circ} \mathrm{C}$ is higher than $50 \%$. Wide environmental tolerance is the prerequisite and guarantee for microorganisms or consortia to be likely applied to further biotransformation.

In nature, lignocellulose is synergistically degraded by a variety of microorganisms [38]. The relationship between strains cannot be competitive when they survive during the establishment of consortiums to ensure that secreted enzymes work together to achieve effective degradation [39, 40]. Cortes et al. (2017) co-cultured lignocellulose-degrading bacteria and fungi by random combination in pairs to enhance their ability to degrade lignocellulose. The results showed that 5 out of 13 different co-culture systems exhibited synergistic effects [41]. This study found that, in the degradation system of consortium 625 , the growth and enzyme activity of strain ZJW- 6 were significantly promoted by strain DA-25 or its filtrate, but those of DA-25 were not promoted by ZJW-6, indicating that ZJW-6 and DA-25 worked in a subordination manner with ZJW-6 as predominant bacteria rather than in a synergistic manner. Karthika screened Acinetobacter baumannii with high lignocellulose-degrading performance from earthworm compost, but its degradation rate was only $45 \%$ [42]. This study also showed that strain DA-25 had a lower degradation rate of lignocellulose. However, Matti et al. found that this type of strain plays a role in removing compounds (which inhibit microbial enzyme activity) produced during the pretreatment of lignocellulosic materials[43], and is often selectively used in the pretreatment process of lignocellulose degradation. Therefore, we speculate that ZJW- 6 may be inhibited by a certain compound while DA-25 or its secretion inhibits this compound when consortium 625 degrades lignocellulose to increase the enzyme activity of ZJW-6 and enhance the degradation of lignocellulose. The detailed mechanism of consortium 625 for enhancing the degradation rate of lignocellulose still needs further research.

\section{Conclusion}

In this study, we screened out Cellulomonas ZJW-6, a strain with the highest lignocellulose degradation rate, from 27 strains previously screened by the research group. The enzyme activity determination showed that it had lower $\beta$-glucosidase activity. Then we used 14 additional strains with higher $\beta$ glucosidase activity than ZJW-6 to establish microbial consortia by combining them with ZJW-6, respectively. The results showed that microbial consortium 625 composed of Acinetobacter DA- 25 and ZJW- 6 had a significantly higher lignocellulose degradation rate than ZJW-6, and a higher level of $\beta$ glucosidase activity. The single factor test indicated that consortium 625 had significantly higher $\mathrm{pH}$ and temperature tolerance compared with ZJW-6. By response surface analysis, we determined the optimal conditions for degradation of lignocellulose by consortium 625 and its optimal degradation rate was $57.6 \%$. After that, we conducted filtrate induction tests. The results suggested that the filtrate of DA-25 had basically the same effect as DA-25, ZJW- 6 and DA-25 played different roles in consortium 625 - ZJW6 was the dominant strain and DA-25 secreted filtrate to promote the growth of ZJW- 6 leading to an increase in the degradation rate. 
Consortium 625 has a strong ability to degrade untreated lignocellulosic materials in nature, and high tolerance to the environment, with further potential for biotransformation applications.

\section{Methods}

\section{Lignocellulosic material and preparation of culture medium}

The rice straws were taken from the rice test field (E125.41, N43.80) of Jilin Agricultural University, cut into sections of about $5 \mathrm{~cm}$, and air-dried to form a lignocellulosic material.

The culture media used in this experiment include: (1) LB medium: $10 \mathrm{~g} / \mathrm{L}$ tryptone, $10 \mathrm{~g} / \mathrm{L}$ sodium chloride, $5 \mathrm{~g} / \mathrm{L}$ yeast extract; $15 \mathrm{~g} / \mathrm{L}$ agar powder was added when preparing a LB solid medium. (2) Liquid culture medium for lignocellulose degradation: $10 \mathrm{~g} / \mathrm{L}$ lignocellulosic material, $10 \mathrm{~g} / \mathrm{L}$ peptone, $5 \mathrm{~g} / \mathrm{L}$ beef extract, $4 \mathrm{~g} / \mathrm{L}$ ammonium sulfate, $2 \mathrm{~g} / \mathrm{L}$ dipotassium phosphate, and $0.5 \mathrm{~g} / \mathrm{L}$ magnesium sulfate heptahydrate. All the culture media above were sterilized at $121^{\circ} \mathrm{C}$ for 30 min before use.

\section{Establishment of microbial consortia and determination of degradation rate}

The 15 strains used in this experiment are lignocellulose-degrading strains screened in the laboratory at the early stage. The establishment of microbial consortia by combining ZJW- 6 with the other 14 strains based on the principle of complementation of enzyme activity is as follows:

Pick a single colony to $10 \mathrm{ml}$ LB liquid medium and culture it at $30^{\circ} \mathrm{C}$ with shaking (160rpm) for $16-24 \mathrm{~h}$. Use an ultraviolet spectrophotometer (Bio-Spes Mini, Shi-madzu, Japan) to determine OD600nm $=0.8$ to prepare a bacteria pre-culture solution (the same below). Inoculate the prepared pre-culture solution into $100 \mathrm{ml}$ lignocellulose degradation liquid medium with an inoculation volume of $1 \%$ and an inoculation ratio of $1: 1$, culture at $30^{\circ} \mathrm{C}$ with shaking (160rpm) for $96 \mathrm{~h}$, centrifuge at $5000 \mathrm{rpm}$ for $10 \mathrm{~min}$, discard the supernatant, repeat washing 3-5 times with distilled water, air-dry to constant weight, and calculate the degradation rate of lignocellulose. The formula is as follows: Lignocellulose degradation rate $(\%)=($ dry weight before treatment - dry weight after treatment) / dry weight before treatment ${ }^{\star} 100 \%$. All experiments were repeated three times.

\section{SrRNA identification of strains and building of phylogenetic tree}

The bacterial genomic DNA extraction kit (provided by Tiangen Biotech (Beijing) Co., Ltd.) was used to extract DNA. Universal primers 27F (5'-AGAGTTTGATCATG

GCTCAG-3') and 1492R (5'-ACGGTTACCTTGTTACGACTT-3') were used to amplify bacterial 16S rRNA gene sequences. $25 \mu \mathrm{L}$ reaction system and amplification conditions: $94^{\circ} \mathrm{C} 4 \mathrm{~min}, 94^{\circ} \mathrm{C} 30 \mathrm{~s}, 55^{\circ} \mathrm{C} 30 \mathrm{~s}$, $72^{\circ} \mathrm{C} 2 \mathrm{~min}, 30$ cycles; $72^{\circ} \mathrm{C} 10 \mathrm{~min}$. The PCR products were detected by $1 \%$ agarose gel electrophoresis, and the DNA recovery kit (provided by Takara Biotechnology (Dalian) Co. Ltd.) was used to recover the 
amplified products. The PCR products were sent to Sangon Biotech (Shanghai) Co., Ltd. for sequencing. The $16 \mathrm{~S}$ rDNA nucleic acid sequence of the strain was compared with the sequences of Acinetobacter and Cellulomonas strains selected in the NCBI database in the Bioedit software, and the comparison result was in the MAGE X 10.2.5 software using the Neighbour Joining method To construct a phylogenetic tree, the booststrap value is 1000 .

\section{Single factor test and response surface optimization design}

Taking the degradation rate of lignocellulose as an indicator, single factor tests were conducted to study the degradation rate of lignocellulose by ZJW- 6 and DA-25 consortium (hereinafter referred to as consortium 625) under the culture conditions (after 96h) of nitrogen sources (peptone, yeast extract, ammonium chloride, ammonium sulfate, urea), nitrogen source addition $(6 \mathrm{~g} / \mathrm{L}, 8 \mathrm{~g} / \mathrm{L}, 10 \mathrm{~g} / \mathrm{L}, 12 \mathrm{~g} / \mathrm{L}$, $14 \mathrm{~g} / \mathrm{L}), \mathrm{pH}(5,6,7,8,9,10)$, temperature $\left(25^{\circ} \mathrm{C}, 28^{\circ} \mathrm{C}, 31^{\circ} \mathrm{C}, 34^{\circ} \mathrm{C}, 37^{\circ} \mathrm{C}\right)$ and inoculation ratio $(4: 1,3: 1$, $2: 1,1: 1,1: 2,1: 3,1: 4$ for ZJW-6 : DA-25).

The optimal conditions for lignocellulose degradation were selected based on the single factor tests. According to Box-Behnken design principle, four factors affecting the degradation rate were screened, namely nitrogen source addition, $\mathrm{pH}$, temperature and inoculation ratio, to conduct tests for four-factor three-level response surface analysis. Design-Expert 10.0.3 was used for statistical analysis of experimental data.

\section{Preparation of filtrate in induction test and determination of lignocellulose degradation rate}

The strains ZJW- 6 and DA-25 with an amount of $1 \%$ of the bacteria pre-culture solution were added to the straw degradation liquid fermentation medium, respectively, and cultured at $30^{\circ} \mathrm{C}$ with shaking (160rpm). When the concentration of the bacterial solution reached $O D=1$, the bacterial solutions were filtered by $0.22 \mu \mathrm{m}$ filter membrane (Millex-GP, Merck Millipore, US) to obtain ZJW-6 and DA-25 filtrates, respectively. $1 \%$ ZJW-6 bacterial solution and 10\% DA-25 filtrate or 1\% DA-25 bacterial solution and $10 \%$ ZJW- 6 filtrate were simultaneously added to $100 \mathrm{ml}$ straw degradation liquid fermentation medium for culture. The determination of lignocellulose degradation rate in the induction test is the same as that in Sect. 2.2.

\section{Determination of strain growth}

The growth of strains ZJW- 6 and DA-25 in consortium 625 lignocellulose-degrading liquid medium was determined using dilution coating method. The pre-cultured bacteria solution was inoculated into $100 \mathrm{ml}$ lignocellulose-degrading liquid medium for culture according to the optimized conditions. $1 \mathrm{ml}$ of culture solution was taken at $6 \mathrm{~h}, 12 \mathrm{~h}, 18 \mathrm{~h}, 24 \mathrm{~h}, 30 \mathrm{~h}, 36 \mathrm{~h}, 42 \mathrm{~h}$, and $48 \mathrm{~h}$, respectively, and diluted by 10 -fold gradient with normal saline. $100 \mu$ l bacterial solution was coated to the LB solid medium, and cultured at $30^{\circ} \mathrm{C}$ for $24 \mathrm{~h}$. The colony-forming unit (CFU) was counted with three repetitions, then the average value was calculated. 
According to the filtrate preparation and induction test methods in Sect. 2.5, the effects of ZJW-6 filtrate and DA-25 filtrate on the growth of strain DA-25 or ZJW-6 in the straw degradation liquid fermentation medium were determined respectively. The time and method for CFU counting are the same as those above.

\section{Determination of enzyme activity}

The enzyme activity of consortium 625 in Sect. 2.6 as well as enzyme activities of endoglucanase [24], xylanase (EC3.2.1.8) [25], lignin peroxidase (EC1.11.1.13) [26], laccase (EC 1.10.3.2) [26], manganese peroxidase (EC 1.11.1.14) [27] and $\beta$-glucosidase (EC 3.2.1.4) [28] in the filtrate induction tests were determined. The definition of activity unit of each enzyme is as follows:

One unit $(U)$ of endoglucanase activity is defined as the amount of enzyme required for production of 1 $\mu \mathrm{mol}$ glucose by degrading cellulose sodium per min per $\mathrm{ml}$ of enzyme solution.

One unit $(U)$ of xylanase activity is defined as the amount of enzyme required for release of $1 \mu \mathrm{mol}$ xylose by catalysis per min per $\mathrm{ml}$ of enzyme solution.

One unit $(U)$ of lignin peroxidase activity is defined as the amount of enzyme that causes a change in UV absorption at $465 \mathrm{~nm}$ within one min.

$\mathrm{U} / \mathrm{ml}=\Delta \mathrm{OD} / \varepsilon$

$\triangle \mathrm{OD}$ : Change in the reaction solution absorbance value at $465 \mathrm{~nm}$ within one min;

$\varepsilon$ : molar extinction coefficient $\left(\varepsilon 465=12000 \cdot \mathrm{M}^{-1} \cdot \mathrm{cm}^{-1}\right)$

One unit $(U)$ of laccase activity is defined as the amount of enzyme required for oxidation of $1 \mu \mathrm{mol} A B T S$ per min per $\mathrm{ml}$ of enzyme solution.

$\mathrm{U} / \mathrm{ml}=\left(\Delta \mathrm{OD} \cdot \mathrm{V}_{1}\right) /\left(\mathrm{V}_{2} \cdot \varepsilon \cdot 10^{-6}\right)$

$\triangle \mathrm{OD}$ : Change in the reaction solution absorbance value at $420 \mathrm{~nm}$ within one min; $\mathrm{V}_{1}:$ Total reaction volume $(2.5 \mathrm{~mL}) ; \mathrm{V}_{2}$ : Enzyme solution volume $(0.5 \mathrm{~mL})$;

$\varepsilon$ : Molar extinction coefficient $\left(\varepsilon 420=36000 \cdot \mathrm{M}^{-1} \cdot \mathrm{cm}-1\right)$

One unit $(U)$ of $\beta$-glucosidase activity is defined as the amount of enzyme required for production of $1 \mu \mathrm{mol}$ pNP by hydrolyzing PNPG at $405 \mathrm{~nm}$ in $1 \mathrm{~min}$.

\section{Statistical analysis of data}

Excel was used for data arrangement, spss was used for significance and correlation analysis, and Graphpad 8.0 was used for graphing.

\section{Nucleotide sequence accession number}


The nucleotide sequence data from strain ZJW-6 and DA-25 based on partial 16S rDNA gene sequences in this study has been submitted to GenBank with accession number EU854302 and MZ206117.

\section{Declarations}

\section{Ethics approval and consent to participate}

Not applicable

\section{Competing interests}

The authors declare that they have no competing interests.

\section{Authors'contributions}

Yunpeng Guan designed and constructed the experiments, participated in the sequence analyses and drafted the manuscript. Hongyun Zhu participated in the enzyme activity determination of this research. Yuan Zhu participated in the filtrate preparation and degradation rate determination in the induction test. Hemei Zhao and Jian Song participated in growth measurement and response surface analysis.Xue Yang and Zhihai Wu participated in the preliminary preparations for this study and the univariate analysis test.Meiying Yang and Lei Wu participated in the design and coordination of the trial and revised the manuscript.All authors read and approved the final manuscript.

\section{Funding}

This study was supported by the Science and Technology Development Plan Project of Jilin Province (2020040316SF) and the "Thirteenth Five-Year Plan" Science and Technology Project of the Education Department of Jilin Province (JJKH20200335KJ).

\section{Acknowledge}

We are grateful for the equipment support of the College of Life Sciences, Jilin Agricultural University.

\section{Consent for publication}

The submitting author certifies that all coauthors have seen a draft copy of the manuscript and agree with its contents for publication.

\section{Availability of data and material}


All data generated or analysed during this study are included in this published article and its supplementary information files.

\section{Figures}

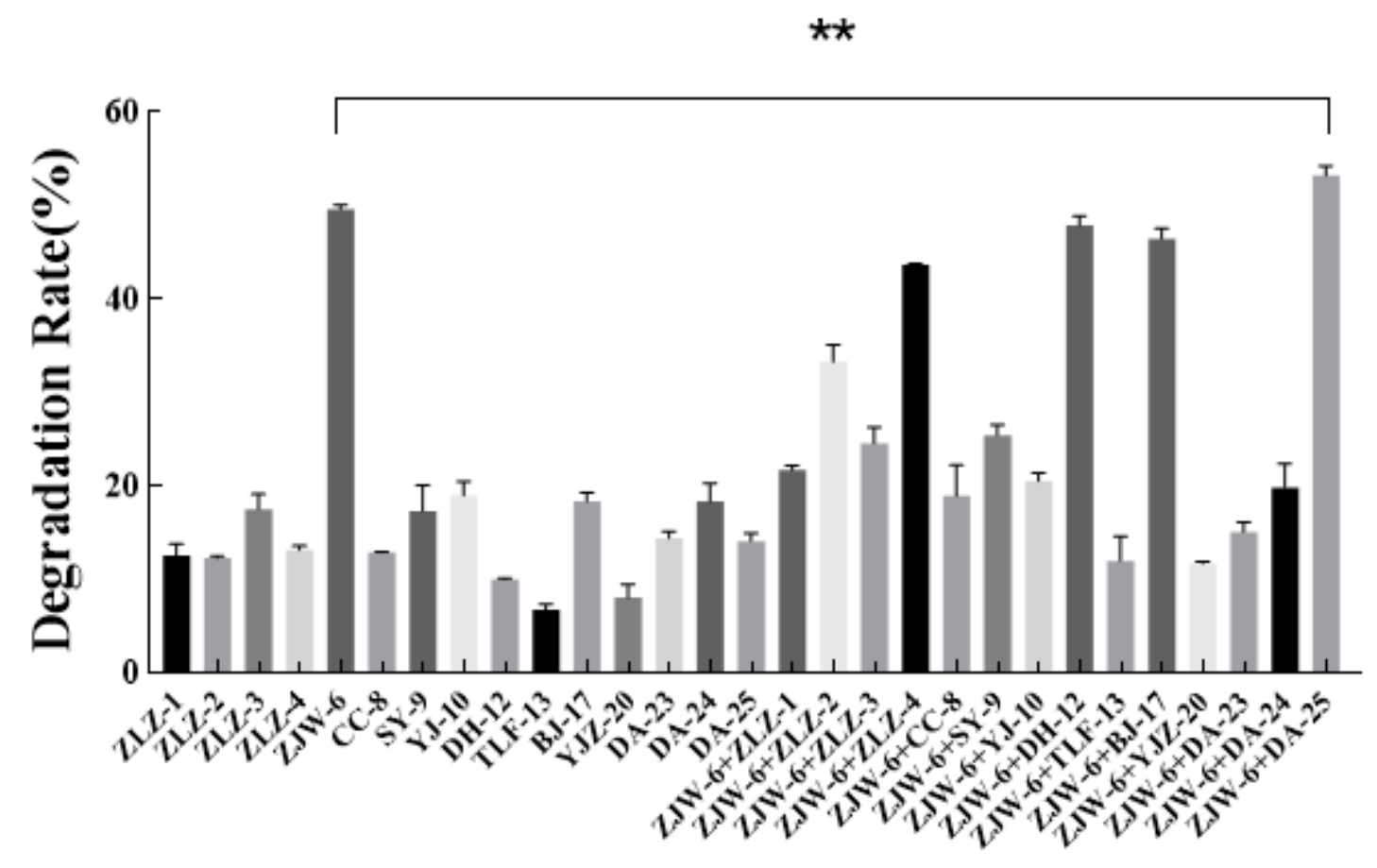

Lignocellulosic

\section{Figure 1}

Degradation rate of lignocellulosic in different lignocellulosic degrading bacteria and composition thereof after 96 hours. (Significant differences between the ZJW-6 and ZJW-6+DA-25 (t-test, P $\leq 0.01$ ) shown by**.) 


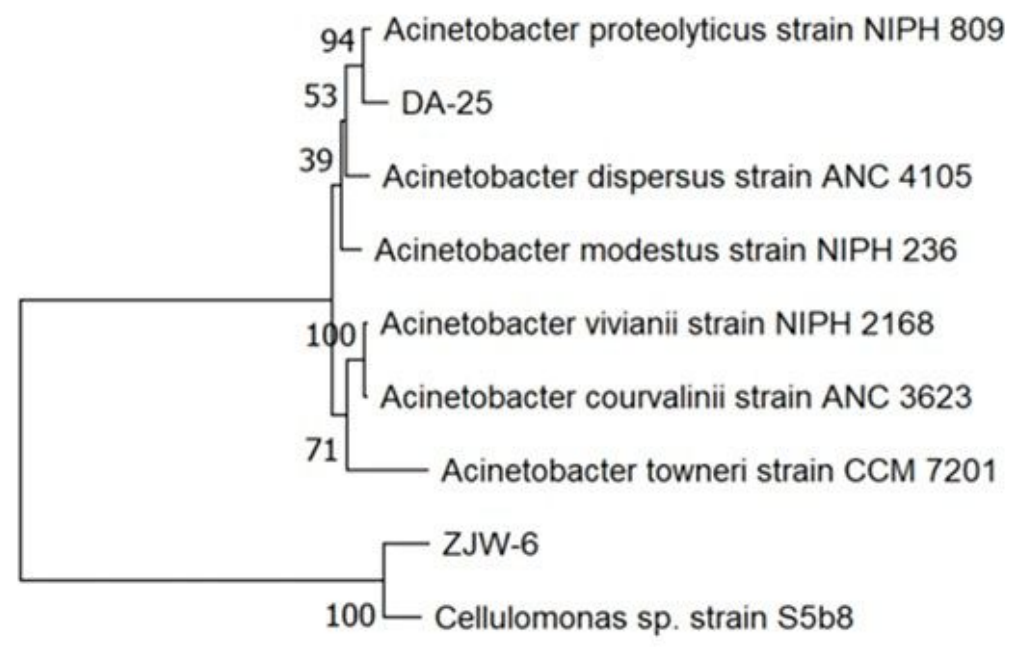

0.02

Figure 2

Molecular Phylogenetic analysis result by NeighbourJoining method

A

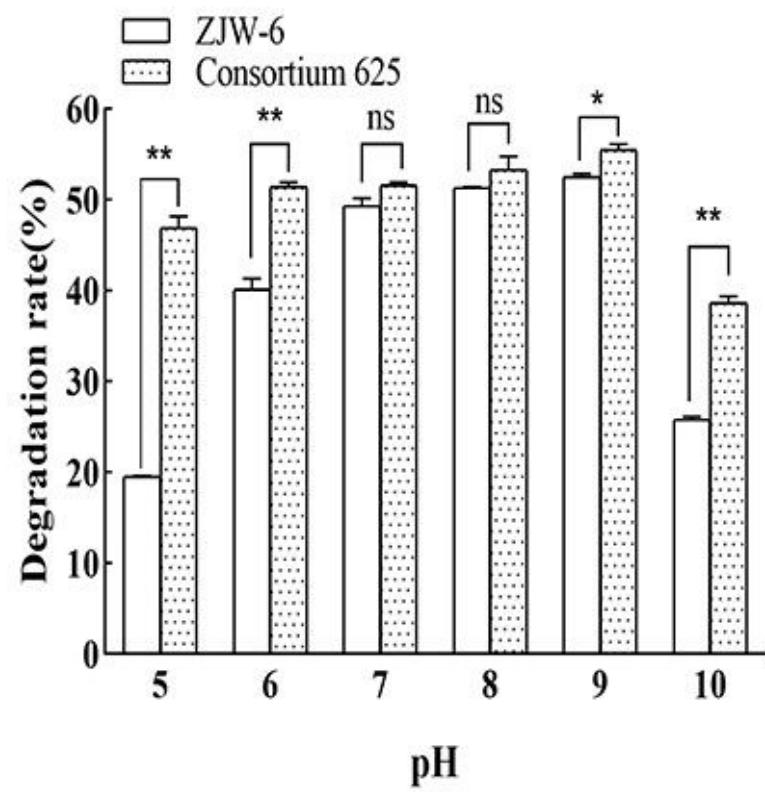

B

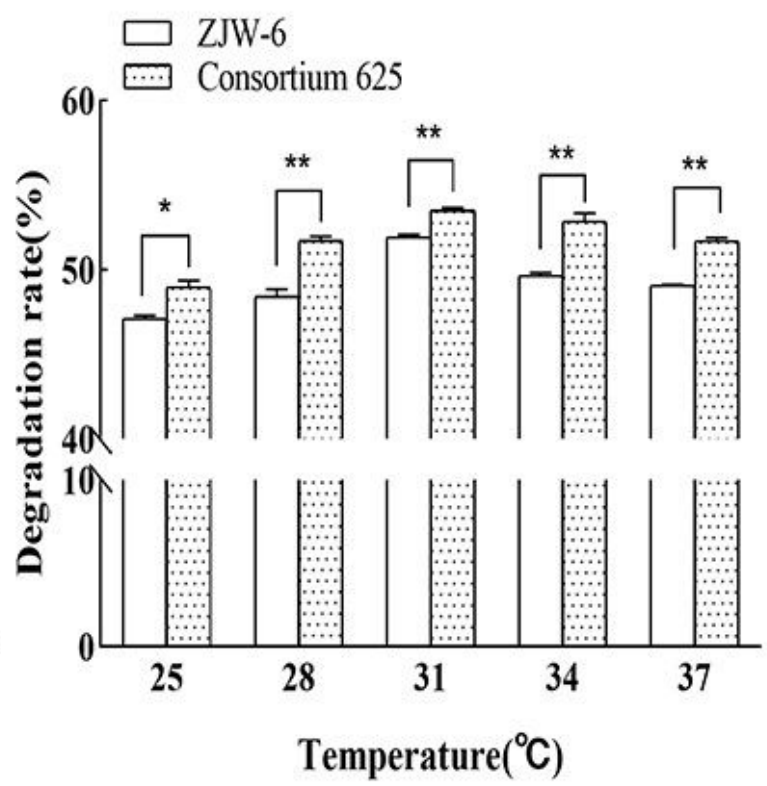

Figure 3

Single factor test results of $\mathrm{pH}$ and temperature of microbial consortium 625. 

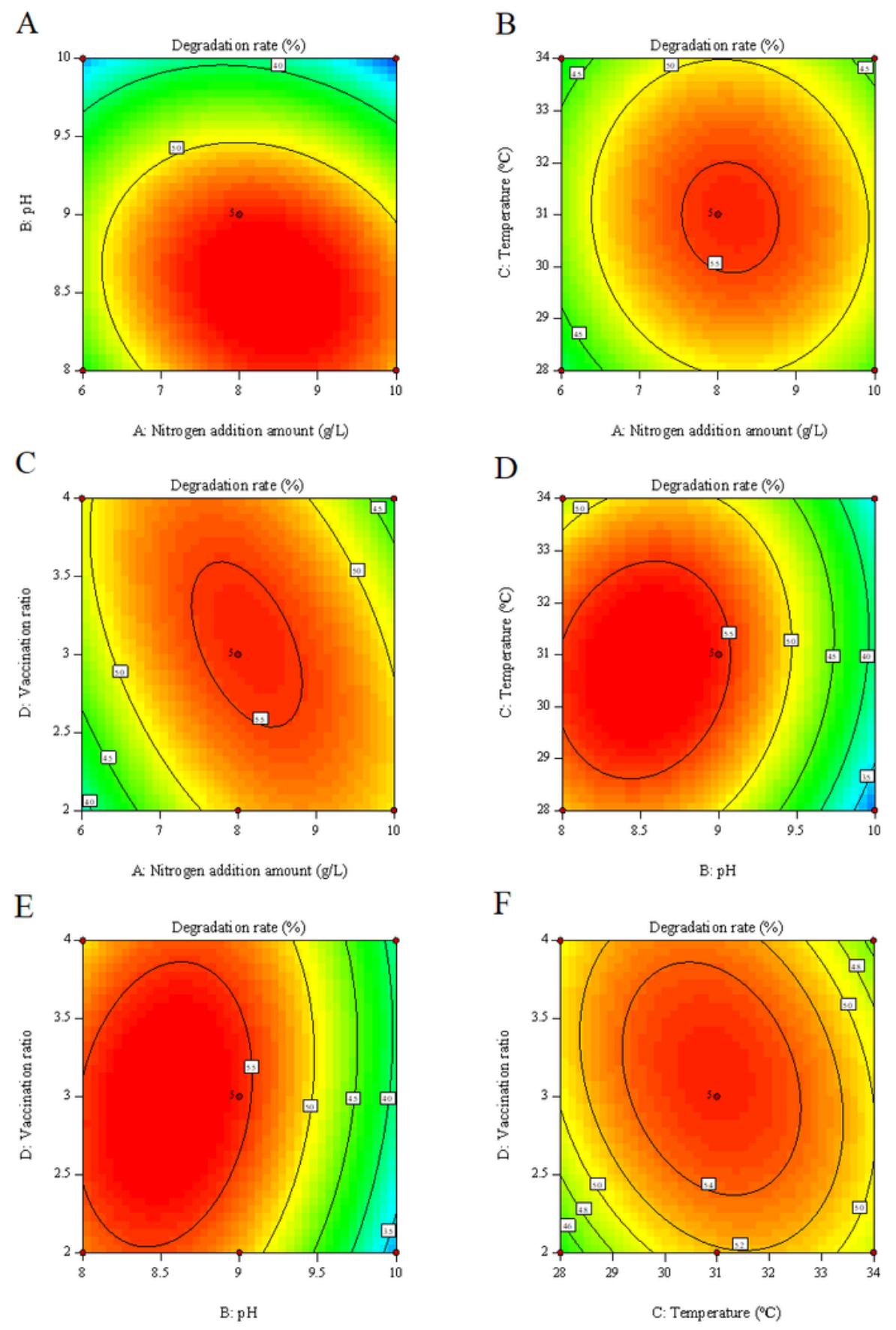

\section{Figure 4}

Response surface optimization effect diagram considering the amount of nitrogen source, $\mathrm{pH}$, temperature and inoculation ratio 
A

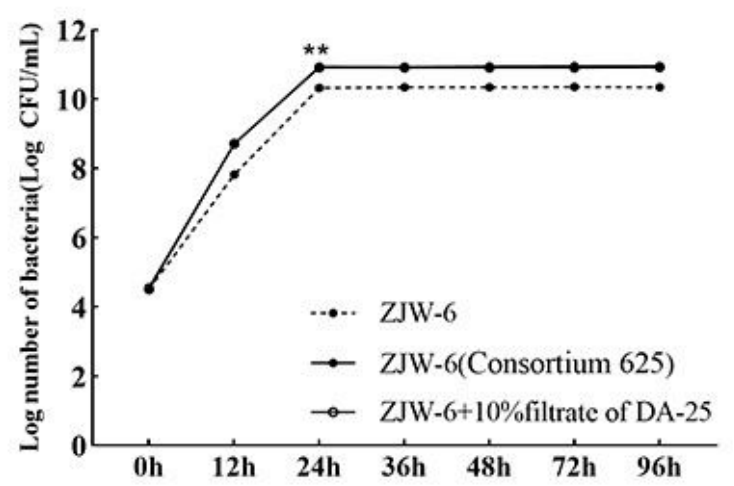

B

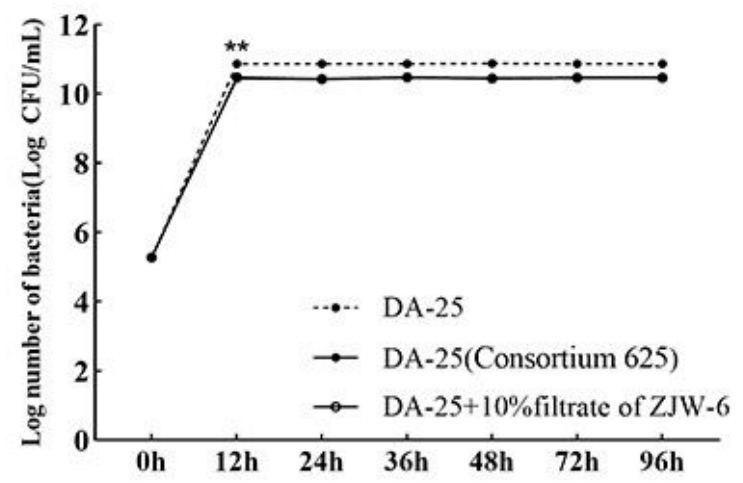

\section{Figure 5}

The growth rate of ZJW- 6 and DA-25 in consortium 625 or induction test 


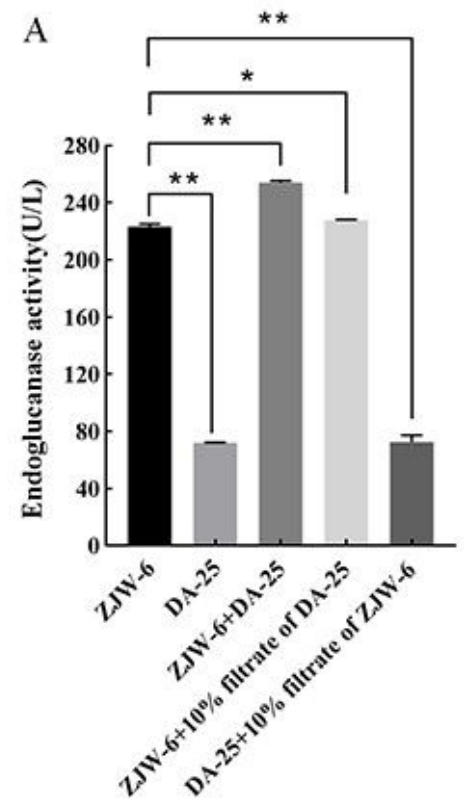

B

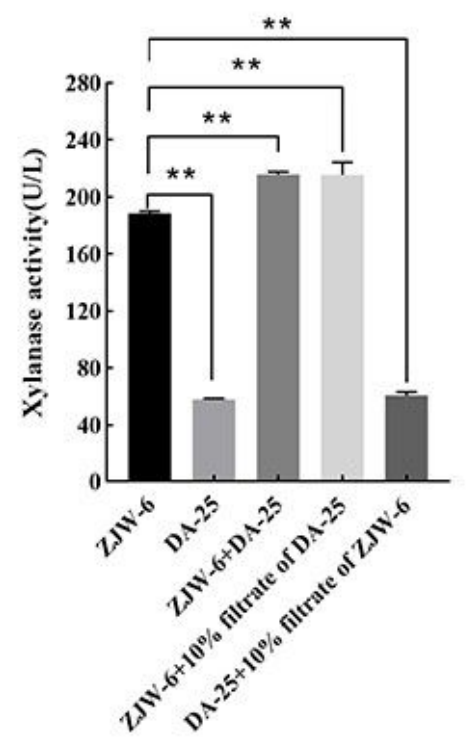

D

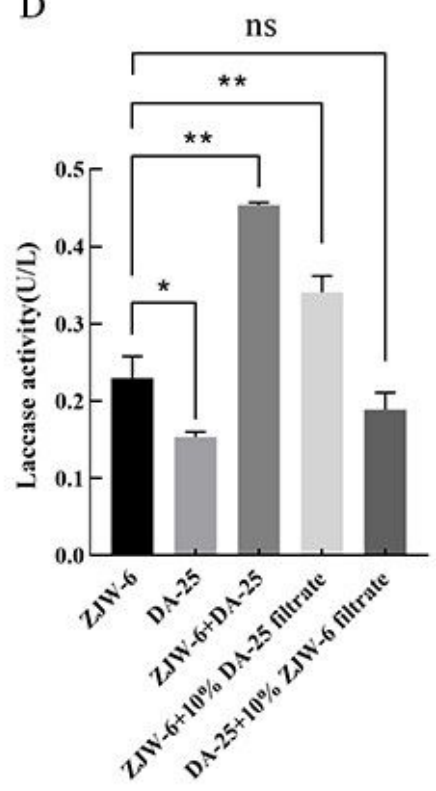

E

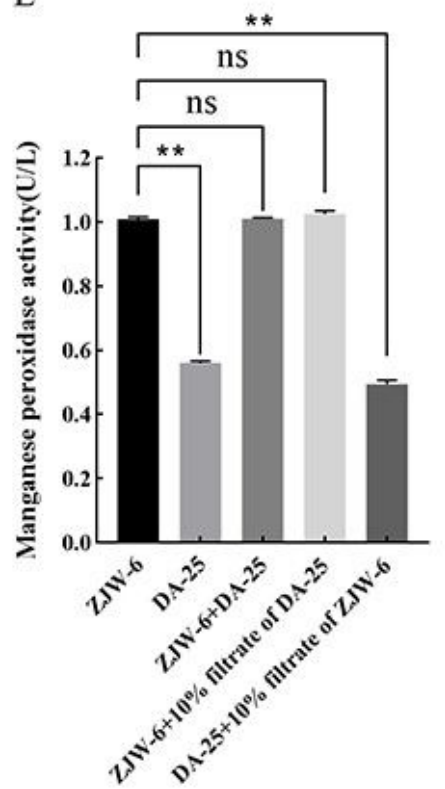

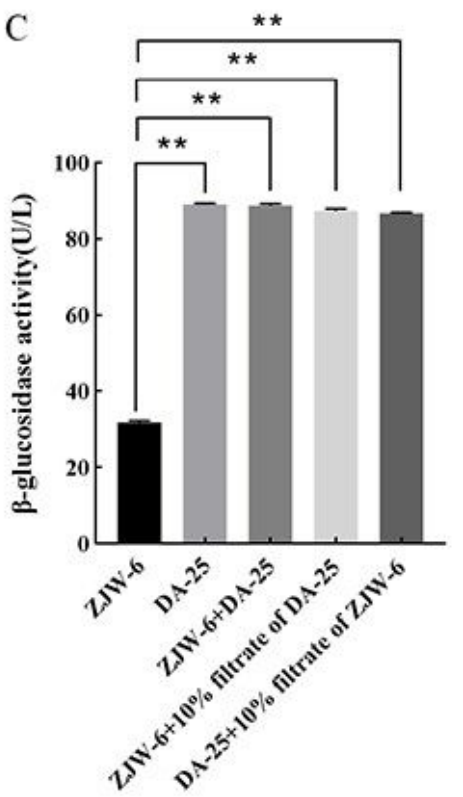

F

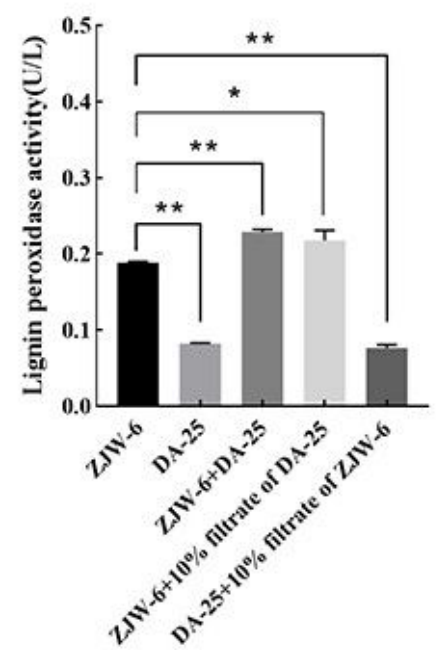

Figure 6

Determination of Key Enzymes for Degrading Lignocellulose after 96 h. Note: A. Endoglucanase activity. B. Xylanase activity. C. $\beta$-Glucosidase activity. D. Laccase activity. E. Manganese peroxidase activity. F. Lignin Peroxidase activity. 


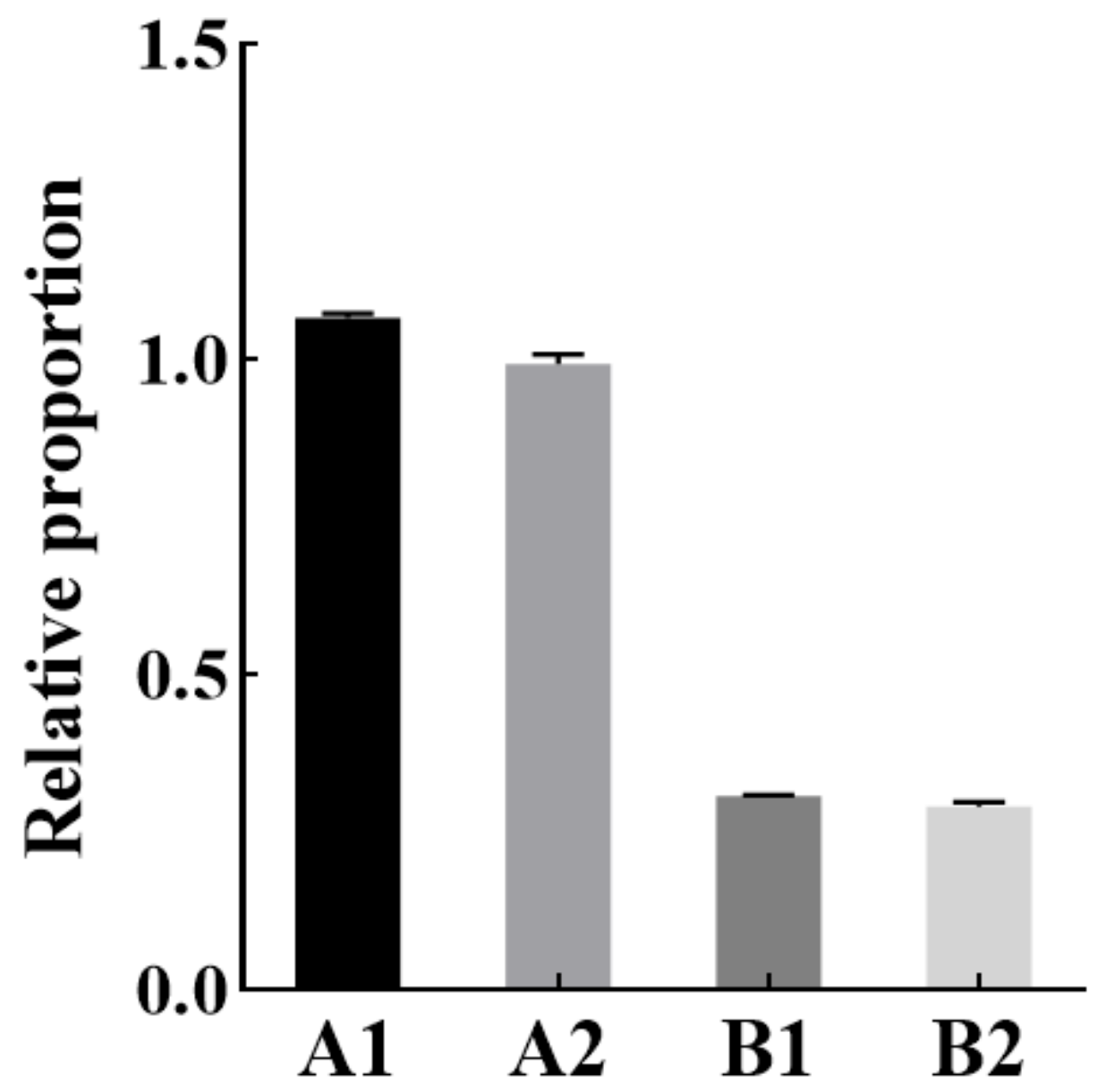

Figure 7

Relative proportional of Degradation rate of lignocellulosic in induction test with consortium 625 and ZJW-6 or DA-25. Note\A1.囚ZJW-6+10\%filtrate of DA-25) / ZJW-6; A2. (ZJW-6+10\%filtrate of DA-25) / Microbial Consortium 625; B1. (DA-25+10\%filtrate of ZJW-6) / ZJW-6; B2. (DA-25+10\%filtrate of ZJW-6) / Microbial Consortium 625

\section{Supplementary Files}

This is a list of supplementary files associated with this preprint. Click to download.

- Fig.S1.tif

- Fig.S2CE.tif 